\title{
Duplication of referral, a tsunami of paper: how much does it cost the Irish health services?
}

\author{
Nicola Walsh ${ }^{1}$ - Lisa Malone ${ }^{1} \cdot$ Sally Ann Lynch ${ }^{1}$
}

Received: 26 September 2021 / Accepted: 18 November 2021 / Published online: 10 January 2022

(c) The Author(s), under exclusive licence to Royal Academy of Medicine in Ireland 2021

\begin{abstract}
Background Prolonged waiting lists increase costs as medical problems may become more expensive to fix. There are also hidden financial costs. Irish Clinical Genetic services have long out-patient waiting times. We noticed duplicate referrals (patients on the waiting list) being re-referred because the patient still had not been seen. These re-referrals waste consultant and administrative time, pose a clinical risk by distracting clinician time, and are costly to our health service.

Methods We prospectively collected duplicate referral data over a 3-month period (1 October 2020-31 January 2021) in order to estimate costs. We costed (1) referring consultant and administrative time; (2) stationary, postage, and storage cost; and (3) receiving consultant and administrative time processing these referrals.

Results We noted 82/986 (8\%) referrals to our service over the trial period were duplicate. The mean length of time between first and duplicate referral was 306 days. In 35/82 (42.68\%), a duplicate referral had already been received (e.g. 3rd or more referral for same patient). In total, we received 132 re-referral letters for 82 patients. Duplicate referrals changed triage outcome in $7 / 82(8.54 \%)$ cases.

Conclusion National Treatment Purchase Fund data suggests that 271,560 patients are waiting $>12$ months for both in- and out-patient public appointments on 1 January 2021. Assuming duplicate referrals are occurring across the Irish health system with equal frequency after 12 months of waiting (8\% of total appointments), then we estimate a conservative cost of 757,392 $€$ per quarter to the health service and an annual cost to the HSE of 3,029,568 €.
\end{abstract}

Keywords Cost · Duplicate referrals · Waiting list

\section{Introduction}

Health care spending in relation to excess patient wait times has been estimated in relation to patient costs (reduced economic activity), caregiver costs, and health care systems costs including excess tests and procedures, inappropriate courses of medication and extraneous medical appointments [1-3]. The interrogation of health care systems costs accrued by long waiting times focus on direct patient interaction with medical professionals and increased patient specific costs. The economic burden of excess waiting times on clinician to clinician communications has not been published in the literature to date. Anecdotally, we noted large volumes of duplication of referrals to our service. Any time spent dealing with these re-referrals is concerning as it diverts

Nicola Walsh

nwalsh4@tcd.ie

1 CHI @ Crumlin, Crumlin, Dublin, Ireland the consultants and administration time away from other patients, gives limited benefit to the re-referred patient, and therefore, poses a clinical risk in addition to a financial cost.

The Clinical Genetic service in the Irish Republic is understaffed in comparison to European norms, and waiting lists are prolonged with routine appointment up to 3 years and priority appointment up to 18 months [4, 5]. In addition, traditional large Irish family sizes has compounded waiting times; as new genes were identified so the demand for cascade testing increased exponentially and as the numbers of at risk relatives requesting cascade genetic testing is $>3$ times higher in Ireland than a family from England, Scotland or Wales, the service was grappling with an overwhelming demand with one-fifth of the normal UK centre staff complement [6].

Anecdotally, we were observing receipt of numerous rereferrals of patients that were already on our waiting list. Each referral has to be triaged and signed by the consultant and processed by the administrative team. Each letter becomes part of the patient record and collectively this will 
increase storage costs. Moreover, the referring medic wastes time dictating another letter and the referring administrative team wastes time re-dictating and absorbing the stationary, postage and storage costs.

As these are time consuming to manage, we audited the volume of re-referrals over a specified period to estimate costs. We estimated the time spent by receiving consultant and administration processing them and estimated the referring clinicians and administrative time and stationary costs. Ultimately, this has a cost to our service, more worryingly dealing with unnecessary paperwork diverts clinician attention away from other patients and is therefore a clinical risk. As it can be expected that other clinicians with long waiting list suffer the same fate, we have extrapolated estimated costs to the wider health service the Health Service Executive (HSE) by using data from the National Treatment Purchase Fund (NTPF) to estimate likely cost annually to the HSE.

\section{Aims}

To estimate the cost of duplicate referrals to a specific health care system, Clinical Genetics. To extrapolate these costs to the HSE overall by using the National Treatment Purchase Fund data to infer numbers of duplicate referrals in the system over a 1-year period. To identify any measure that might reduce referral duplication.

\section{Methods}

We conducted a prospective review of all letters of referral received in a tertiary centre for Clinical Genetics over a period of three months (1 November 2020-31 January 2021). We identified duplicate referrals on patients who had previously been accepted to our waiting list but the appointment was still pending. We defined duplicate referrals as a letter received with the intention of referring a patient where they have already been accepted to the waiting list. These duplicate referrals contained no additional clinical or demographic relevant information.

Duplicate referrals were further subdivided into two distinct categories: (1) consultant (more complex) clinic letter where all patient information elicited at that clinic visit was included and may have been directed to more subspecialities and (2) direct (more simple) letter where we received a letter directed only to clinical genetics requesting an outpatient clinic appointment.

We reviewed these charts in order to identify any previous referrals (e.g. triplicate or more) and have included this extra correspondence in our costings.
Exclusion criteria We excluded inappropriate referrals to our service that were re-directed elsewhere. We excluded patients who were re-referred who had been previously seen but discharged from our service (no longer on the waiting list). We excluded correspondence that notified us of a change in patient details (e.g. change of address, new pregnancy, etc.).

Data from all referrals eligible for inclusion in our study was extracted including: number of referrals to our service on the same patient whilst the patient had still not been seen, re-referral clinician(s), re-referral date(s), and cost of stationary and postage. Thereafter, we sourced the family chart and conducted analysis of previous referrals including initial date that the patient or family in question was referred to our service, the initial referral clinician and if and how many other letters had been received in the interim time frame. We also identified if any change in triage decision making occurred on the basis of re-referral.

We analysed the time spent on specific facets of the triage process experienced by both consultant and administrative workers. We estimated the time required for a referral letter to be dictated, typed and posted by the referring team and the time spent triaging and filing each referral by the receiving team. This aspect was calculated by performing a time analyses of the administrative tasks involved via observation of one of our experienced administrative team who timed herself typing up 10 letters and the average sum of 10 processes was calculated. We used this to cost the time spent by the referring team. We estimated the time spent triaging patient referrals on receipt of the letter by the receiving team (time analysis by author, SAL) and time processing the referral by the administrative team (time analysis by author, LM) in order to estimate costs by the receiving team. We also conducted analysis of postal costs as an electronic referral process was not available and all the duplicate referrals were received by post. Initial estimates were on the costs 82 letters received during the 3-month audit. We then separately analysed costs for the total re-referrals $(n=132)$ in this cohort ( 82 re-referrals plus all other re-referrals found within this group).

Using our data, we calculated a conservative estimate of possible excess correspondence that occurs annually within the HSE.

\section{Results}

We received eighty two $(n=82)$ duplicate referral letters out of a total 986 letters received (8\%) that were eligible for inclusion in our study between 1 November 2019 and 31 January 2020 . The majority, $72 / 82$ (88\%), were in the paediatric age group. The mean number of days elapsed between 
Table 1 Cost analysis of 82 letters received during the trial period. We calculated salary costs based on Type A Consultant on a HSE public contract. The differing costs for consultant referrals, includ- ing administrative time, was to differentiate letters that were solely re-referring the patient from those received that were letters directed towards multiple clinicians but included re-referral request to genetics
Referring clinician dictation: Consultant (direct [simple] referral): $4 \mathrm{~m}(n=45 ; € 6.8 € /$ letter)

Consultant (clinic [more complex] letter): $5 \mathrm{~m}(n=37 ; € 8.5 € /$ letter) $\begin{array}{ll}\text { Consultant triage: } & \text { Time spent: } \\ 8 \mathrm{~m}(n=82 ; € 13.6 € / \text { letter }) & 1,021 \mathrm{~min}\end{array}$

$12.5 \mathrm{~min} /$ letter

Cumulative cost:

$€ 1,735.7$

$€ 21.2 € /$ letter initial and subsequent duplicate referral received during the audit period was 378 days (range 8-1748 days). However, the time lapse in cases where only one duplicate $(35 / 82)$ had been received (excluding the cases where the re-referral was a triplicate or more) was 306 days. The mean number of days between initial referral and duplicate referral received during the study period, where more than two referrals had been received, was 949 days (2.6 years).

Only one duplicate was referred both initially and subsequently by a general practitioner. A further referral had been referred by a consultant and a duplicate referral received later from the patients GP. The rest, 80/82, both initial and subsequent referrals, were all consultant to consultant referrals.

Duplicate referrals changed the patient's outcome (change in triage decision from routine to priority appointment) in $8.54 \%$ of cases $(n=7)$. In $91.46 \%$ of cases $(n=75)$, triage remained unchanged following receipt of duplicate referral.

Thirty five re-referral letters eligible for inclusion in our study had already been preceded by 50 additional duplicate referral letters in the time elapsed between initial referral and the dates in which we conducted our study. Of note, two patients were referred 5 times and one patient was referred 6 times. In total, 132 duplicate referrals were noted on these 82 patients. The mean number of extra correspondences received on the 82 patients, not including initial referral is 1.6 letters per patient.

The time analysis revealed that the mean time calculated for hospital consultant dictated clinic letter is $5 \mathrm{~min}$. We received Consultant Clinic letters (more complex) letters for 37 duplicate referrals that were eligible for inclusion in our study. Direct (more simple) letters of referral to the clinical genetics service relating to individual patients $(n=45)$ all originated from

Table 2 Cost analysis of 82 letters received during the trial period. We calculated personnel costs based on HSE the published salaries for Grade V Clerical Officer with 3 years experience. The differing costs for consultant referrals, including administrative time, was to hospital consultant doctors and the mean time calculated for the dictation of standard referral letter is $4 \mathrm{~min}$, see Table 1 .

40 letters were received via national postal service by standard mail at a cost of $1 €$ each.

We timed administrative duties on receiving correspondence within the department. Mean calculated time for the combined activities on receiving post (sorting/delivering incoming post to destination, retrieving patient files, triaging and filing) is 14 min per letter, see Table 2.

Each individual re-referral costs $34.8 €$ per letter received. For the three month duration over which this study took place, the total cost of re-referrals to the health care system is $2852.8 €$. The time spent by clinicians and administrative staff in sending and receiving these letters is $41.5 \mathrm{~min}$ time for each letter received. Estimates of the exponential costs accrued by the multiple referrals is detailed in Table 3.

The National Treatment Purchase Fund still recorded a total of 271,560 patient waiting $>12$ months to be seen in outpatients $(12-15$ months $=54,632,15-18$ months $=45,945$, 18 months $+170,983$ patients) on January 2021. This only reflects out-patient activity. The NTPF also lists a total of 570 patients $(234=12-15$ months, $137=15-18$ months and $199 \geq 18$ months) waiting $>12$ months for an in-patient or day case admission. In total, therefore, there are 272,130 patients waiting $>12$ months for a public appointment or admission to hospital for treatment [7 www.ntpf.ie].

We noted that duplication occurs in $8 \%$ of our cohort and that duplication occurs with a mean time of 306 days. Of the 272,130 patients awaiting HSE appointments $>12$ months, $8 \%$ equates to 21,770 patients. By extrapolation, each duplicate referral received accrued a cost of $34.79 € /$ letter, which suggests a total annual cost to the HSE of 3,029,568 €.

differentiate letters that were solely re-referring the patient from those received that were letters directed towards multiple clinicians but included re-referral request to genetic

\begin{tabular}{|c|c|c|c|c|}
\hline \multirow[t]{2}{*}{ Typing letter: } & $\begin{array}{l}\text { Direct (more simple) referral: } 12 \mathrm{~m} \\
\quad(n=45 ; 4.7 € / \text { letter })\end{array}$ & Postage and stationary: $€ 2.2(n=82)$ & $\begin{array}{l}\text { Receipt of letter (sorting): } \\
14 \mathrm{~m}(\mathrm{n}=82 ; 5.5 € / \text { letter) }\end{array}$ & $\begin{array}{l}\text { Time spent: } \\
2391 \mathrm{~min} \\
29 \mathrm{~min} / \mathrm{letter}\end{array}$ \\
\hline & $\begin{array}{l}\text { Clinic (more complex) letter: } 19 \mathrm{~m} \\
\quad(n=37 ; 7.4 € / \text { letter })\end{array}$ & & & $\begin{array}{l}\text { Cumulative cost: } \\
€ 1,116.7 \\
13.6 € / \text { letter }\end{array}$ \\
\hline
\end{tabular}


Table 3 Table showing extra costs accumulated by patients on waiting lists which was accrued by extra correspondence. The sum of the cost of all extra correspondence received for the 82 patients included

\begin{tabular}{|c|c|c|c|c|}
\hline Total & Dictation and typing & Stationary & $\begin{array}{l}\text { Receiving team triaging and process- } \\
\text { ing by admin }\end{array}$ & Total cost \\
\hline 47 duplicate referrals & $633.80 €$ & $103.40 €$ & $897.70 €$ & $1,634.90 €$ \\
\hline 26 triplicate referrals & $701.24 €$ & $114.40 €$ & $993.20 €$ & $1,808.84 €$ \\
\hline 6 quadruplet referrals & $242.73 €$ & $39.60 €$ & $343.80 €$ & $625.63 €$ \\
\hline 2 quintuplet referrals & $107.88 €$ & $17.60 €$ & $152.80 €$ & $278.28 €$ \\
\hline 1 sextuplet referrals & $67.43 €$ & $11 €$ & $95.50 €$ & $\begin{array}{l}173.93 € \\
4,521.58 €\end{array}$ \\
\hline
\end{tabular}

It was not possible to estimate cost of storage of the excess letters but it is worth noting that 528 letters would fill one of our "genetic boxes," which we use for storage offsite and the cost of storage off site of a genetic box per year is $5.2 €$.

\section{Discussion}

The Clinical Genetic services, chronically under-resourced in Ireland, has had long out-patient waiting lists (up to 3 years for a routine appointment) for years. The issue is of such concern that recently published data detailed the consequences to patients who have died prior to receiving an appointment [8]. As the only Clinical Genetic service in the Republic offering advice on all aspects of Clinical genetics, it does mean there are limited options for patients to be seen elsewhere. A consequence is frustration from both the patient and referring physician that the patient has not been seen in a timely manner. This frustration results in repeated correspondence with limited tangible benefit to the patient. This correspondence is a waste of both human and stationary resources. It poses a clinical risk as it diverts clinician time away from other patients and is of no tangible benefit to the majority of these patients as the triage outcome did not change.

Traditionally, $60 \%$ of referrals to Clinical Genetics are adults; the fact that $88 \%$ of the duplicate referrals occur in the paediatric bracket demonstrates concern by both the parents and clinicians to advocate for their children/patients. Despite this advocacy, clinical triage is based on a clinical need and queue jumping is not permitted within the HSE. Most referrals were already on the priority list and could not be further expediated. The initial triage decision was acceptable in most cases. It was unclear what the rational was to change the small number from routine to priority. It is possible that the receiving clinician assumed family anxiety was high and tried to reduce the waiting times further by expediating the appointment as best they could. We have previously published on the complexity of triage within in our study is $4521.58 €$. This table does not include costs from initial referral and acceptance to a waiting list
Clinical Genetics leading to inconsistencies both within and between clinical genetics units [6]. The triage change could also have been an example of this inconsistency.

On average, our data suggest that our service receives approximately 328 duplicate referrals per annum (82 times 4) on $8 \%$ of all referrals and an additional 200 (50 times 4) triplicate or more referrals, suggesting a total of 528 additional correspondence per annum to our service. At the time of the audit, there were 1900 patient on the consultant clinical genetics waiting list (www.ntpf.ie) and a further 850 on the Genetic counsellor waiting list (not recorded by NTPF), suggesting 3\% $(82 / 12,750)$ of all current referrals that are waiting to be seen result in at least a duplicate or more referral. There were $>900,000$ patients awaiting in and out patient appointments or procedures on the NTPF website in July 2021, if $3 \%$ of them resulted in duplication of referral that would work out at 27,000 unnecessary additional correspondence per annum (www.ntpf.ie). Even if one was to suggest that the duplication occurs after a 1-year wait, with 272,130 waiting $>12$ months, that would equate to 8163 letters annually.

Is our experience likely to be mirrored elsewhere within the HSE? Can we extrapolate up? There is only one public Clinical Genetic centre in the Irish Republic and, unless the patient accesses the cross border care scheme or opt to be seen privately (access to private providers is also limited), they have to wait as there is nowhere else for the patient to be seen. Barriers to transparent referral pathways exist and result in referral duplication as the referring clinician cannot confirm their patient is on a Genetics waiting list. These barriers include individual hospital IT systems and the need to adhere to GDPR regulation maintaining patient privacy. GDPR considers Genetic evaluation and testing in the highest risk bracket, thereby preventing non Genetic staff electronic access to our OPD waiting lists. Other specialists clinics are openly available within $\mathrm{CHI}$ to a referring consultant from within the directorate. Changes to traditional referral pathways, such as online referral pathway, have been shown to improve referral processes in other subspeciality disciplines with long waiting lists [2-4]. However, even in 
countries with a universal health management system, privacy issues preclude access to patient data and knowledge of whether or not your patient is on a specialist list is not immediately accessible. These roadblocks might suggest that even in an efficient system duplicate referrals will continue to occur albeit at a lower rate.

Our data would suggest that most of the duplicate referrals are occurring approximately 10 months (306 days) after the initial referral. If the waiting list could be reduced to 10 months, it is likely that most of this problem could be avoided. We did note a small number of the duplicate referrals were sent by two consultants simultaneously, or two copies sent within weeks of each other by the same consultant suggesting local system inefficiencies.

Clinical Genetics, being a tertiary referral service, receives most of its referrals from other consultants. Only 2/82 duplicate referrals were from GPs. Other services will have differing referrers, many with a higher GP referral base. For specialites with a higher GP referral base, would GPs be more or less likely to re-refer patients? Would they consider trying other care providers for the same service? Either way, even if they are more likely to refer to a second provider, this would result in wastage across the health service as a whole.

Other services will also have better access to alternative similar expertise within other hospitals (public or private) on the island of Ireland or in Northern Ireland via the cross border directive. In other words, there is somewhere else for these patients to go. There is currently only one part-time consultant who provides Clinical Genetics consultations on the island of Ireland. Patients can avail of the cross border directive but many will have a rare disease, which are often chronic disabling disorders and travel is not straightforward. In addition, COVID has significantly reduced cross border referrals. However, it is extremely unlikely that we are the only specialty suffering this unnecessary volume of paperwork. The fact that there are 272,130 patients waiting $>12$ months for public OPD appointments is indicative that a bottleneck in terms of access to specialist services across the country is widespread which, we believe, is likely to result in duplication of referral across many specialites. We suspect this issue is endemic in a system that tolerates chronically long waiting lists and suggests that many patients cannot afford to access alternative private providers and others may not be in a position to travel to elsewhere within Ireland to provide their care publically.

Recruitment of consultants within the Irish Republic is difficult with $>700$ vacant consultant posts nationally [9]. Currently, there are two consultant Clinical Genetic posts which remain unfilled. It is disheartening to have to chronically wade though unnecessary correspondence with no benefit to anyone knowing this is taking time away that would be better spent on patient care. It suggests a multi-faceted dysfunctionality inherent within our health system, poor front line staffing and poor IT systems preventing innovation and more prompt appointments.

Whilst this additional cost is one metric of the dysfunction within our system, the true cost of this dysfunction is experienced by the families. However, staff distress and retention is likely to be a factor in units where the waiting times become insurmountable.

It is important to note that our study was carried out during a strict lockdown due to COVID-19 and the overall referrals were down $3 \%$ indicating that the true cost of referral duplication is likely higher.

\section{Conclusion}

As our study demonstrates, for each referral received and accepted onto a waiting list $>306$ days, we can expect $4.5 \%$ to be duplicated, $2.5 \%$ to be triplicated, and $1 \%$ of referral events will result in even more correspondence.

Long waiting lists contribute to increased morbidity and mortality. They also result in unnecessary paperwork that poses a clinical risk and a cost to a health service. Patient safety and experiences are optimised in efficient healthcare services. Fully integrated national services would be the optimal patient centred model of care. At present, our study suggests that that if we work to build a service that operates efficiently and keeps waiting list times down to $<10$ months, we can not only improve patient care but this will help conserve scarce healthcare resources. We recognise that the cruellest aspect of waiting lists is the adverse effects on patients and their careers in terms of morbidity, mortality and distress. However, our study reveals an important additional aspect in terms of hidden costs that has not been described to date.

\section{Declarations}

Conflict of interest The authors declare no competing interests.

\section{References}

1. O'Reilly J et al (2012) Paying for hospital care: the experience with implementing activity-based funding in five European countries. Heal Econ Policy Law 7:73-101. https://doi.org/10.1017/ S1744133111000314

2. Stokes E, Somerville R (2008) The economic cost of wait times in Canada. Canada Cent Spat Econ Can Med Assoc 1-60

3. Delikurt T et al (2015) A systematic review of factors that act as barriers to patient referral to genetic services. Eur J Hum Genet 23:739-745. https://doi.org/10.1038/ejhg.2014.180

4. Lynch SA, Borg I (2016) Wide disparity of clinical genetics services and EU rare disease research funding across Europe J Community Genet 7(2):119-126. https://doi.org/10.1007/ s12687-015-0256-y 
5. www.hse.ie/eng/staff/leadership-education-development/met/plan/ specialty-specific-reviews (date accessed 01/09/2021)

6. McVeigh TP, Donnelly D, Al Shehhi M et al (2019) Towards establishing consistency in triage in a tertiary specialty. Eur J Hum Genet 27(4):547-555. https://doi.org/10.1038/s41431-018-0322-0

7. www.ntpf.ie/ (date accessed 17th August 2021)

8. Bradley L, Lynch SA (2021) Dying to see you? Deaths on a clinical genetics waiting list in the Republic of Ireland; what are the consequences? J Community Genet 12(1):121-127. https://doi. org/10.1007/s12687-020-00491

9. www.irishexaminer.com/news/arid-40707092.html www.irishexaminer. com/news/arid-40707092.html (date accessed 9th Nov 2021)

Publisher's Note Springer Nature remains neutral with regard to jurisdictional claims in published maps and institutional affiliations. 